\title{
Short-Term Results of Patient Specific Instruments (PSI) - Total Knee Replacement (TKR)
}

Mohamed Mosa Mohamed Mahmoud*1, Amr Abdelhalem Amr ${ }^{1}$, MA Hafez ${ }^{2}$

${ }^{1}$ Department of Orthopaedic and Trauma Surgery, Faculty of Medicine, Al Azhar University, Assiut Branch, ${ }^{2}$ Department of Orthopaedic and Trauma Surgery, Faculty of Medicine, 6 October University

*Corresponding author: Mohamed Mosa Mohamed Mahmoud, Mobile: (+20) 01009092327,

E-Mail drmohamedmosa@yahoo.com

\section{ABSTRACT}

Background: TKR is an effective treatment for severely arthritic knees. PSI TKR is a recent technique, which relies on preoperative formulation of specific instruments for every patient preoperatively, aiming at decreasing surgical time and instruments and increasing accuracy and functional outcome. Objective: Assessment of functional and radiological outcome of PSI TKR after a period of 3 years duration. Patients and Methods: 40 TKRs in 24 patients were done using CT-based PSI technique. Pre-and postoperative knee society score (KSS) were measured and digital long-leg X-rays were obtained for all patients. Hip-knee-ankle, proximal tibia land lateral distal femoral angles together with mechanical axis deviation were measured for all patients to assess the mechanical axis alignment after TKR. Results: HKA (hip knee ankle) has decreased from 4.70 varus preoperatively to 1.27 varus. The mean knee society score had markedly increased from 31.2 preoperatively to 85.3 with fair to excellent KSS was $95 \%$ after 3 years duration. Conclusion: PSI TKR is an effective technique in knee replacement as conventional method. With the advantages of reduction of the operative steps and concurrent potential complications.

Keywords: PSI, TKR.

\section{INTRODUCTION}

Total knee replacement (TKR) is considered as an effective treatment for severely arthritic knees (1). Patient specific instrumentation (PSI) TKR is a recent technique that relies on preoperative $\mathrm{CT}$ (computed tomography) scan or MRI of the arthritic knee ${ }^{(2)}$. Then three-dimensional printing technology used to produce two patient-tailored templates (one tibial and one femoral) ${ }^{(3,2)}$. These templates act as bone cutting guides (for direct bone cutting through the templates) or as pin locator (pin-placement guides) ${ }^{(4)}$.

PSI aims at simplifying the operative technique for TKR through decreasing surgical steps, shortening operative time, avoiding invasion of femoral medullary canal by intramedullary guide, minimizing blood loss and obviating fat embolism, bone injury and possibly the rate of infection ${ }^{(3,5)}$. The aim of this study was to assess short-term functional and radiological outcome of TKR on patients indicated for primary TKR done by PSI using cutting guides templates.

\section{PATIENTS AND METHODS}

During the period from January to October 2017, 40 TKRs in 24 patients were done using CT-based PSI technique in 6 October University Hospital. Average age of patients was $58.9 \pm 9.9$ year $(30-70)$. The patients were divided to 21 females and 3 males.

The preoperative diagnosis was severe osteoarthritis in all patients except 4 patients had rheumatoid arthritis. Preoperative KSS, x-rays and CT scans of the knees had been done.
CT scan was done under a special protocol (it was done in $1 \mathrm{~mm}$ cuts and scanogram from hip to ankle was done in two planes coronal and sagittal planes. Then printed in a computer disc (C.D.).

\section{Preoperative planning:}

Planning began by importing the $\mathrm{CD}$ to a special software in the computer. Firstly, measuring mechanical axis in both coronal and sagittal plane was done (figures 1 and 2). After that, the software reconstructed the knee in three-dimensional images without patella. Then the tibial image was removed and distal femur cut and sizing were done (figure 3), then removal of femur image, and tibial image were brought back for cut and sizing (figure 4). In the virtual cuts, distal femoral cut perpendicular to the mechanical axis of the femur in the coronal plane, and in 3 degrees flexion in the sagittal and in three degrees of external rotation was done. The proximal tibial cut was adjusted perpendicular to the mechanical axis of the tibia and tibial slope was adjusted according to anatomy of the patient or company guidelines. The amount of cut was about $10 \mathrm{~mm}$ of the healthy side but also we could change level of cut as we needed in special circumstances (e.g. flexion deformity). After alignments, cuts and sizing had been done, we had 3-D view of the virtual postoperative knee (figure53).

The surgeon revised the planning at all steps and he could change the planning according to clinical and radiological conditions of the patient (e.g. he could change size, femoral rotation, tibial slope and level of bone cuts). After that, the software produced two 3-D 
images of patient, specific cutting guides (patient specific instruments) for both tibia and femur (figures 5 and 6). After that, two-PSI templates were produced by rapid prototyping machine. These cutting guides contained sites for regular saw blades, sites for pin fixation and lugholes. Side of the knee, size and patient names were engraved on each template. Lastly, the two cutting guides (templates) were received to the hospital, where they were sterilized and packaged. The patient called to be admitted to the hospital at the morning day of surgery.

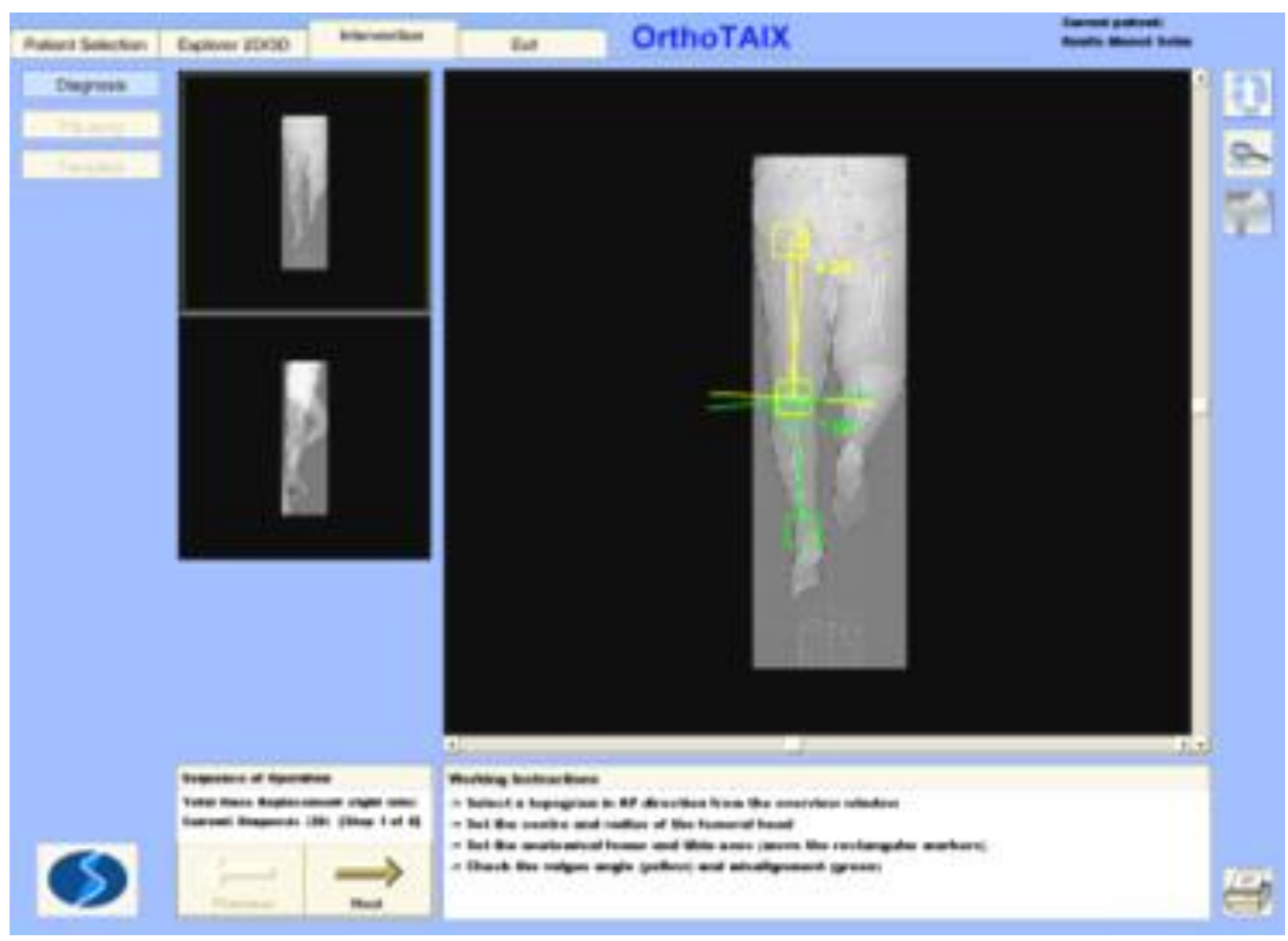

Figure (1): measuring coronal mechanical axis

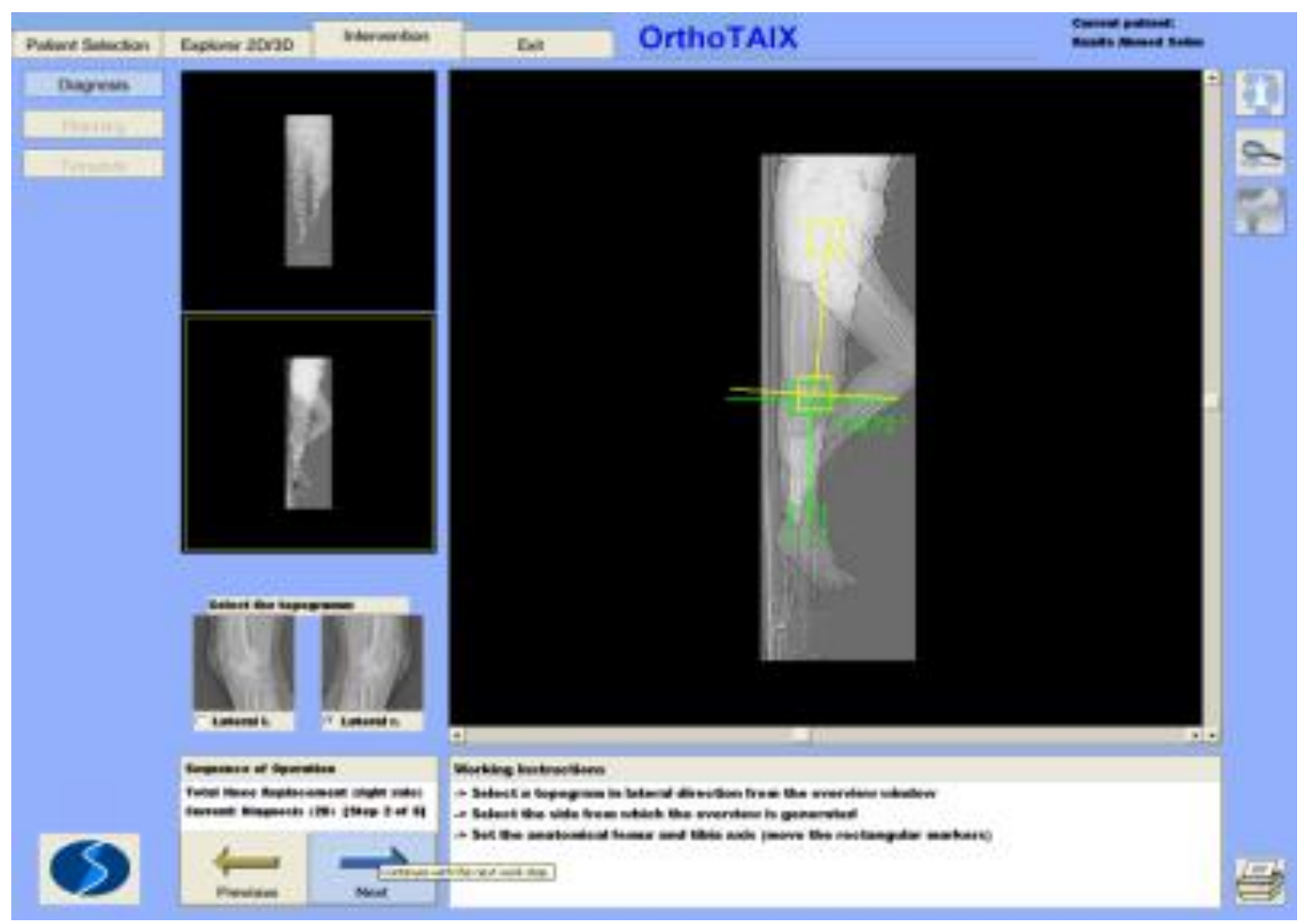

Figure (2): measuring sagittal mechanical axis 

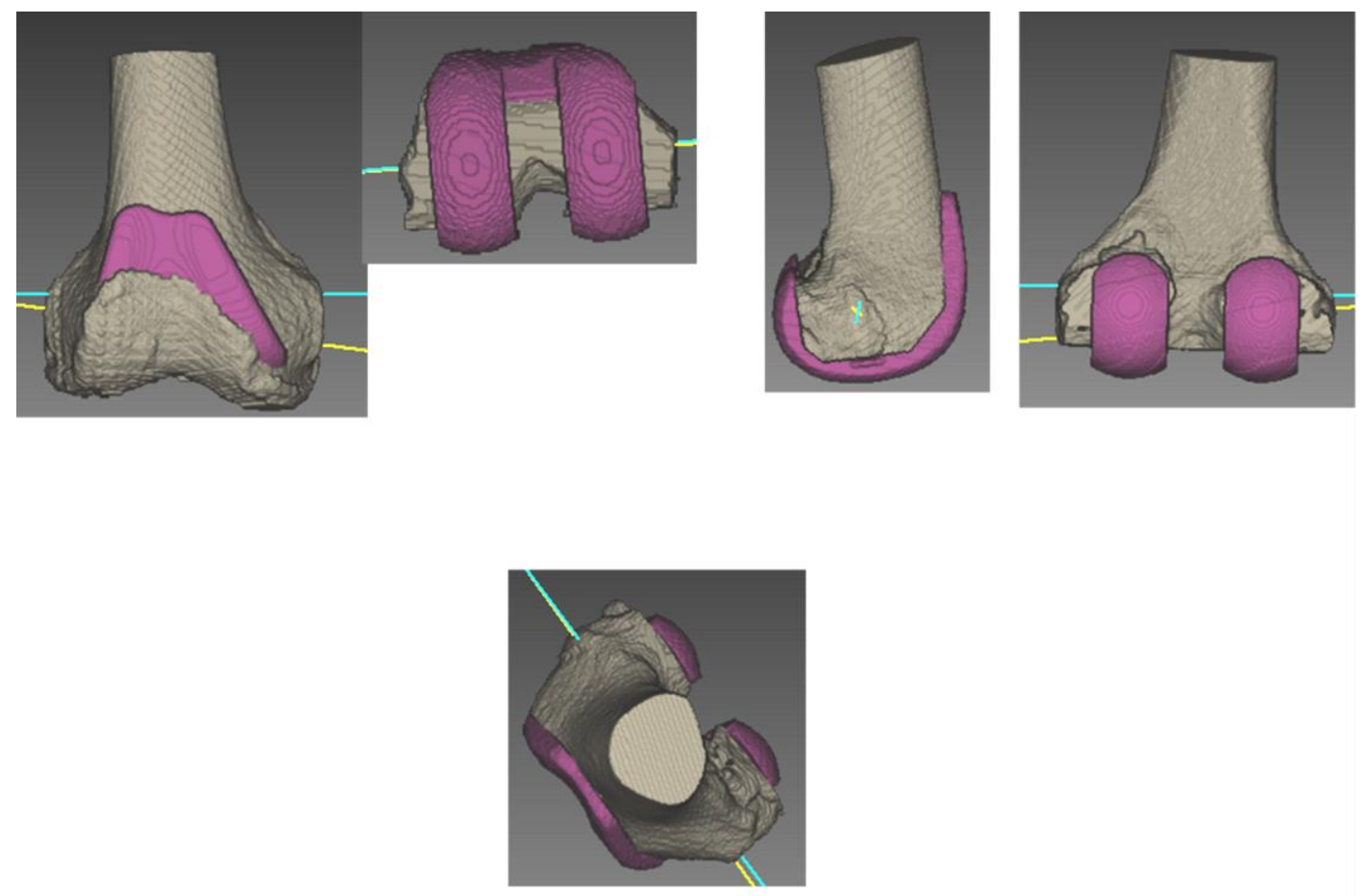

Figure (3): distal femoral cuts and sizing
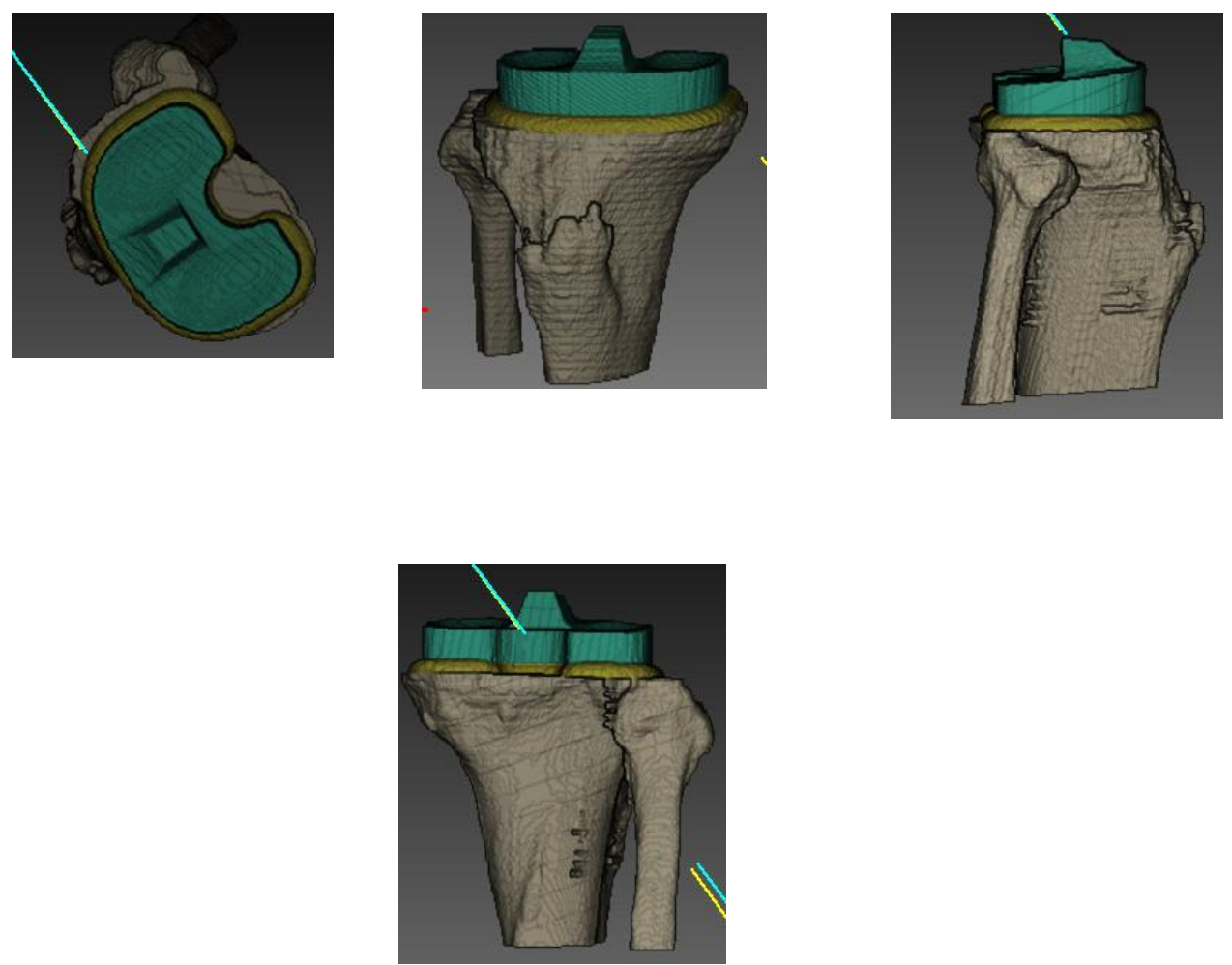

Figure (4): proximal tibial cut 

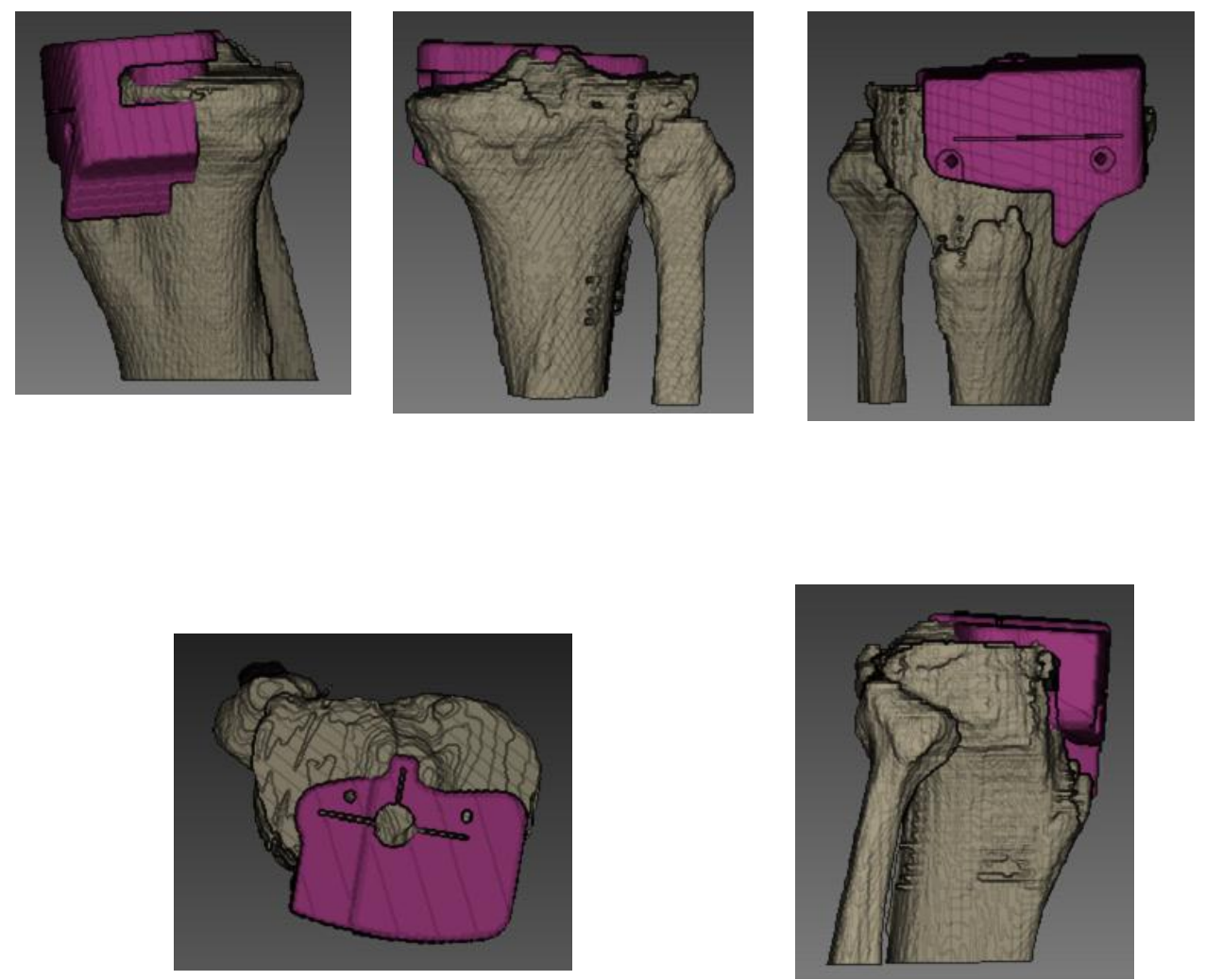

Figure (5): patient specific tibial cutting guide in 3-D image
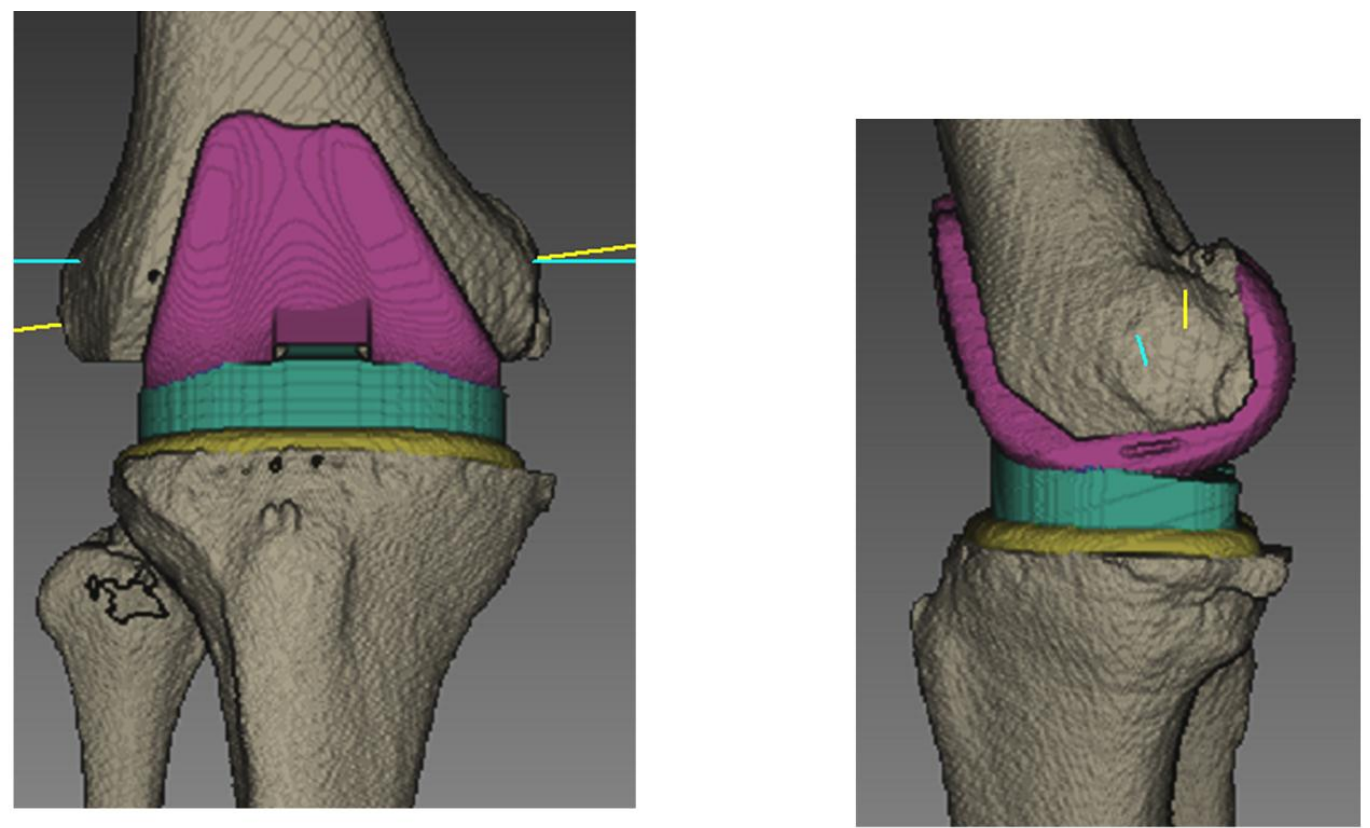

Figure (6): 3-D images of the virtual TKA 


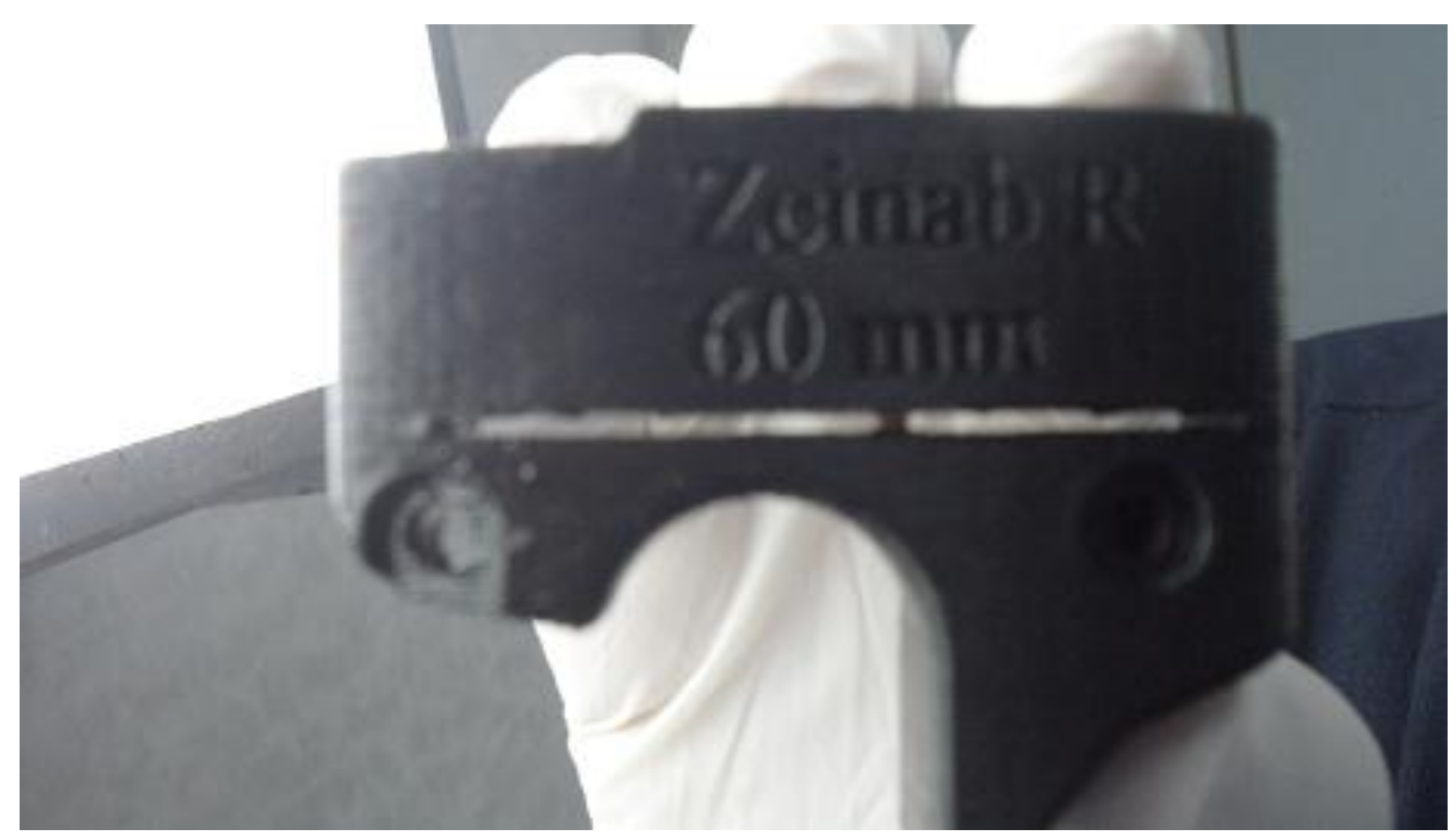

Figure (7): Tibial cutting guide

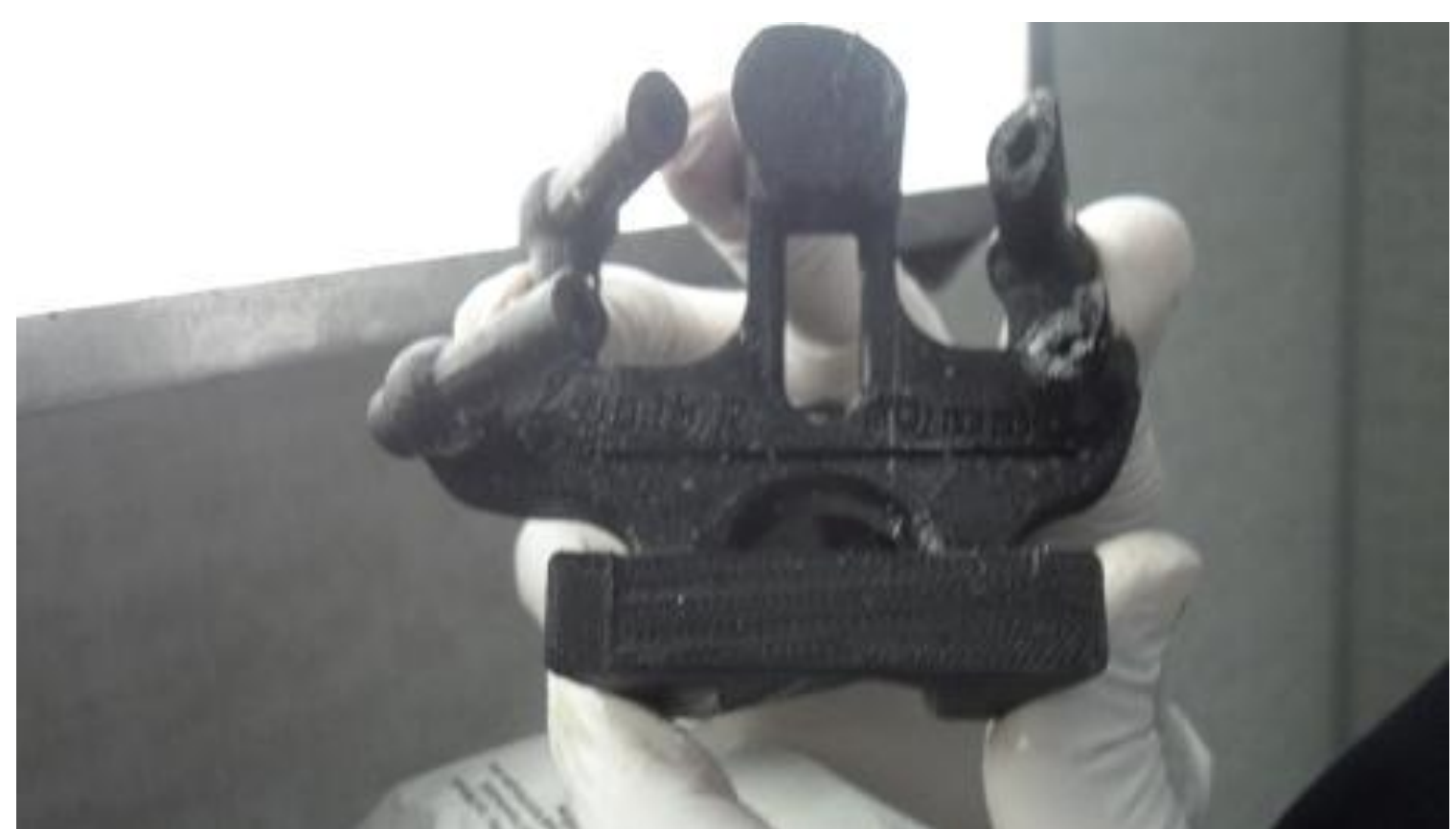

Figure (8): Femoral cutting guide

\section{Surgical technique:}

Medial parapatellar approach had been done for all cases. After god exposure, the femoral cutting guide was fixed to the distal femur and all femoral cuts were done through it (figure 9, 10). Femoral box was done by box osteotome of the company. Then, tibial cut and preparation were done through tibial cutting instrument (fig.11, 12). Trial components and any soft tissue release were done (fig.13). Finally, implantation of the prosthesis and closure were done. 


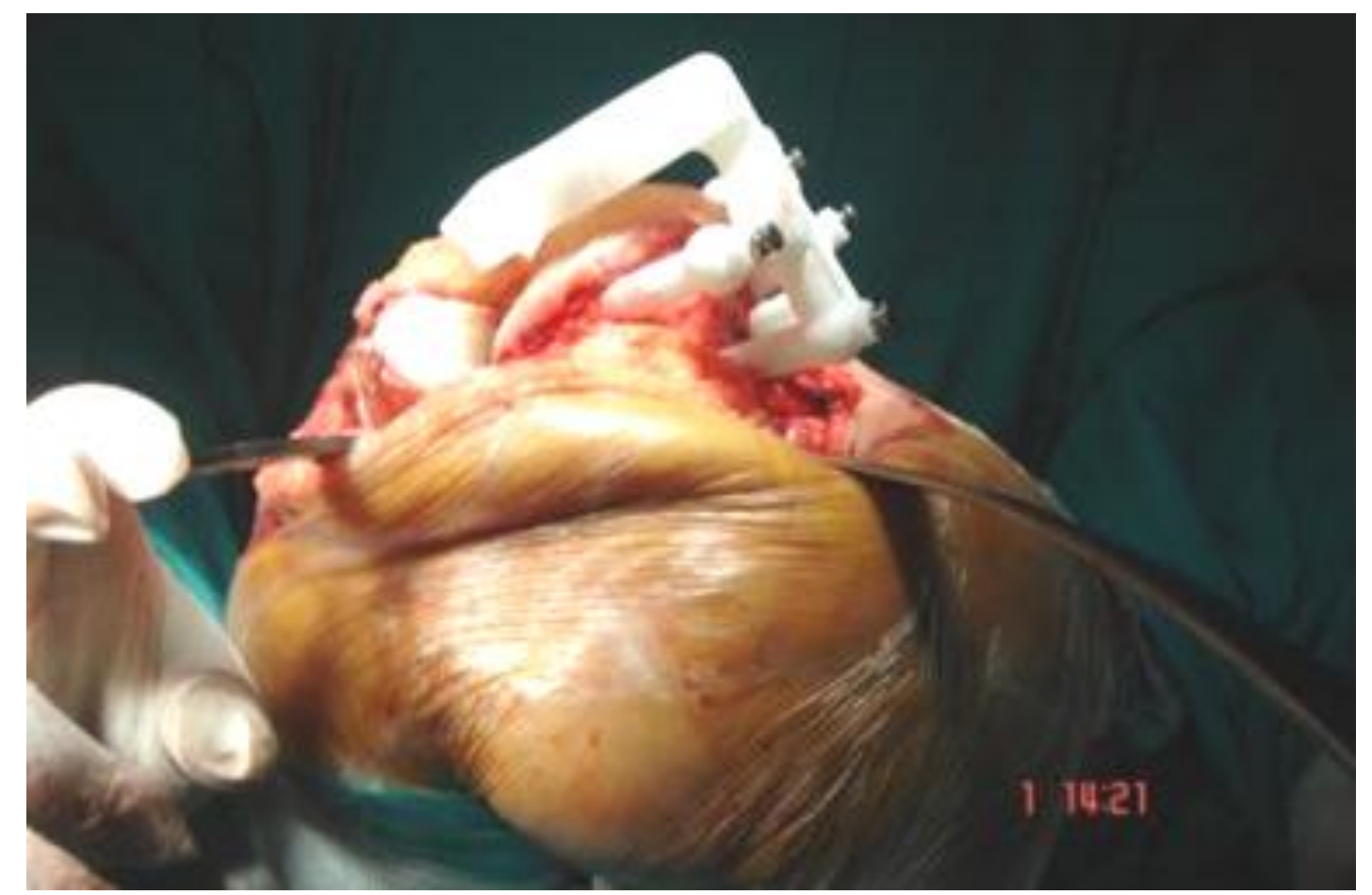

Figure (9): Femoral cutting guide side view

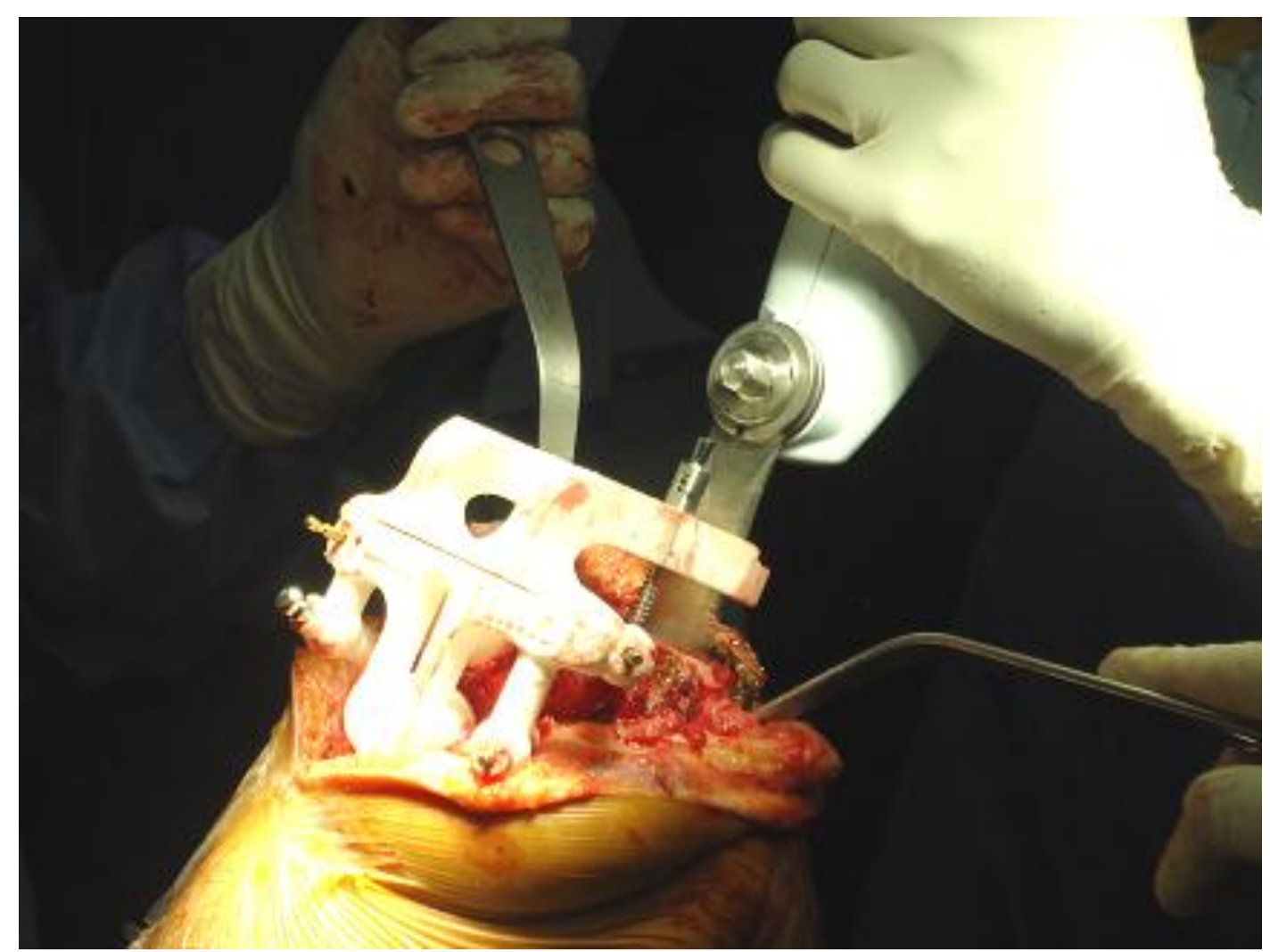

Figure (10): All femoral cuts done through PSI guide 


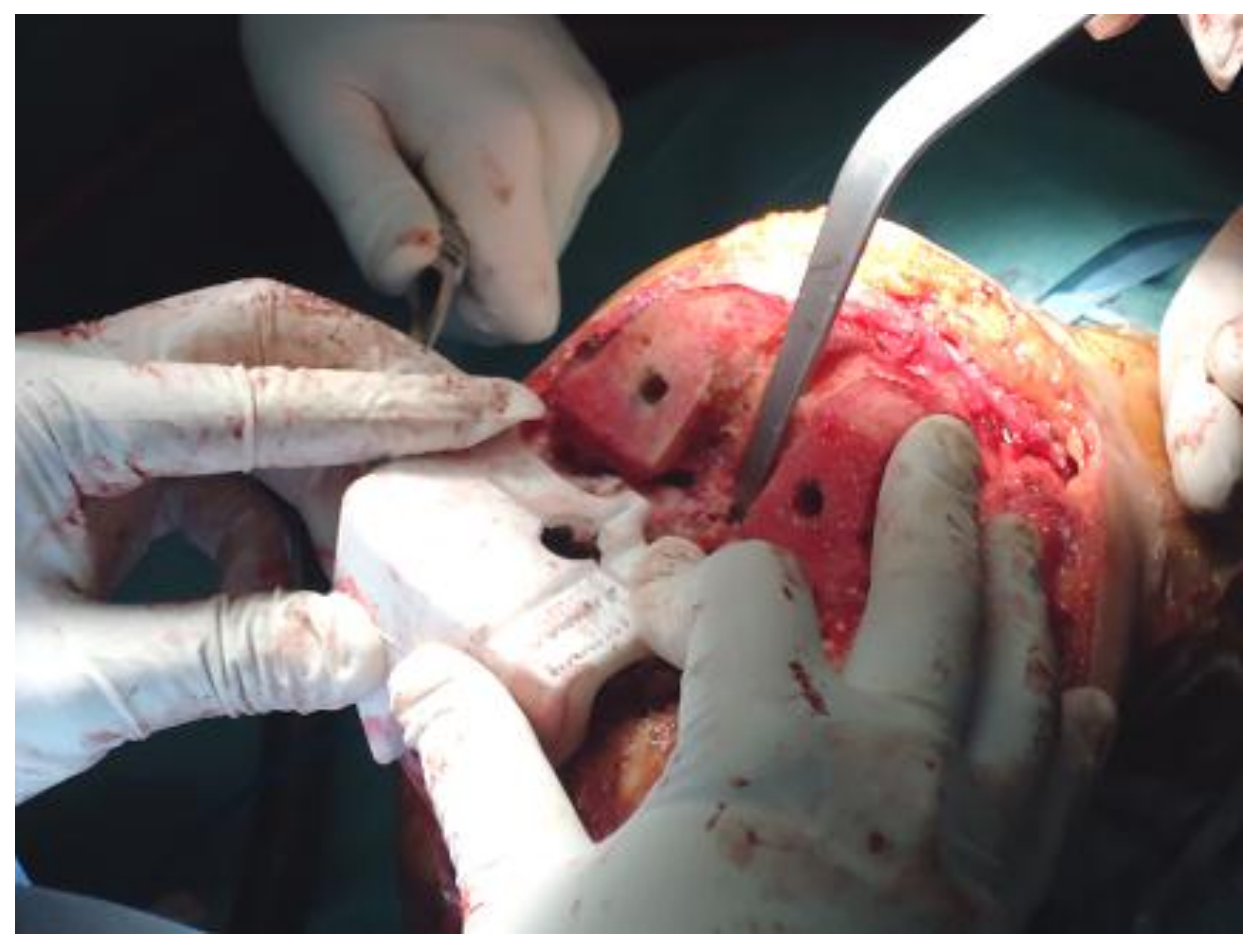

Figure (11): Tibial cutting template fixed on the tibia

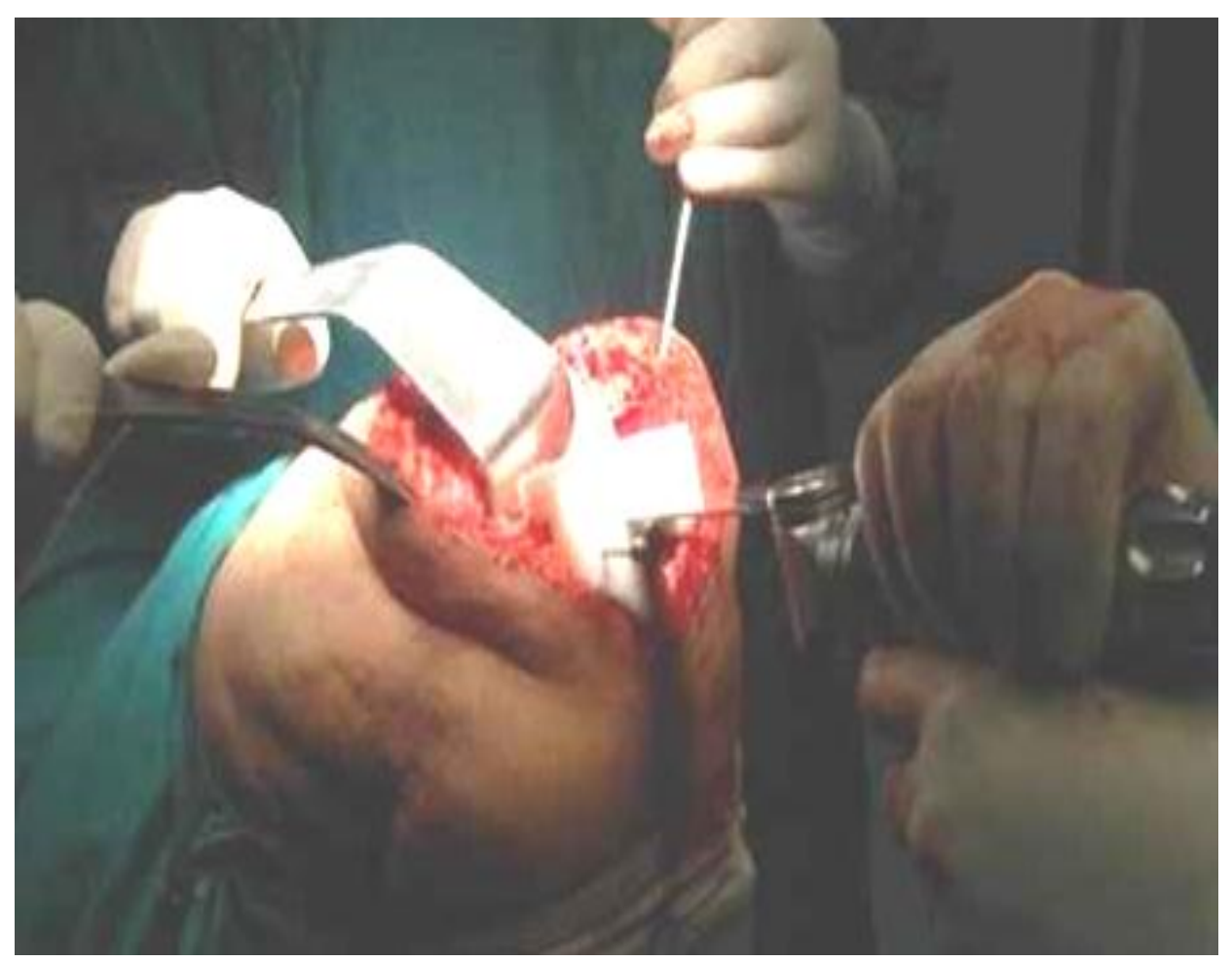

Figure (12): Tibial cut 


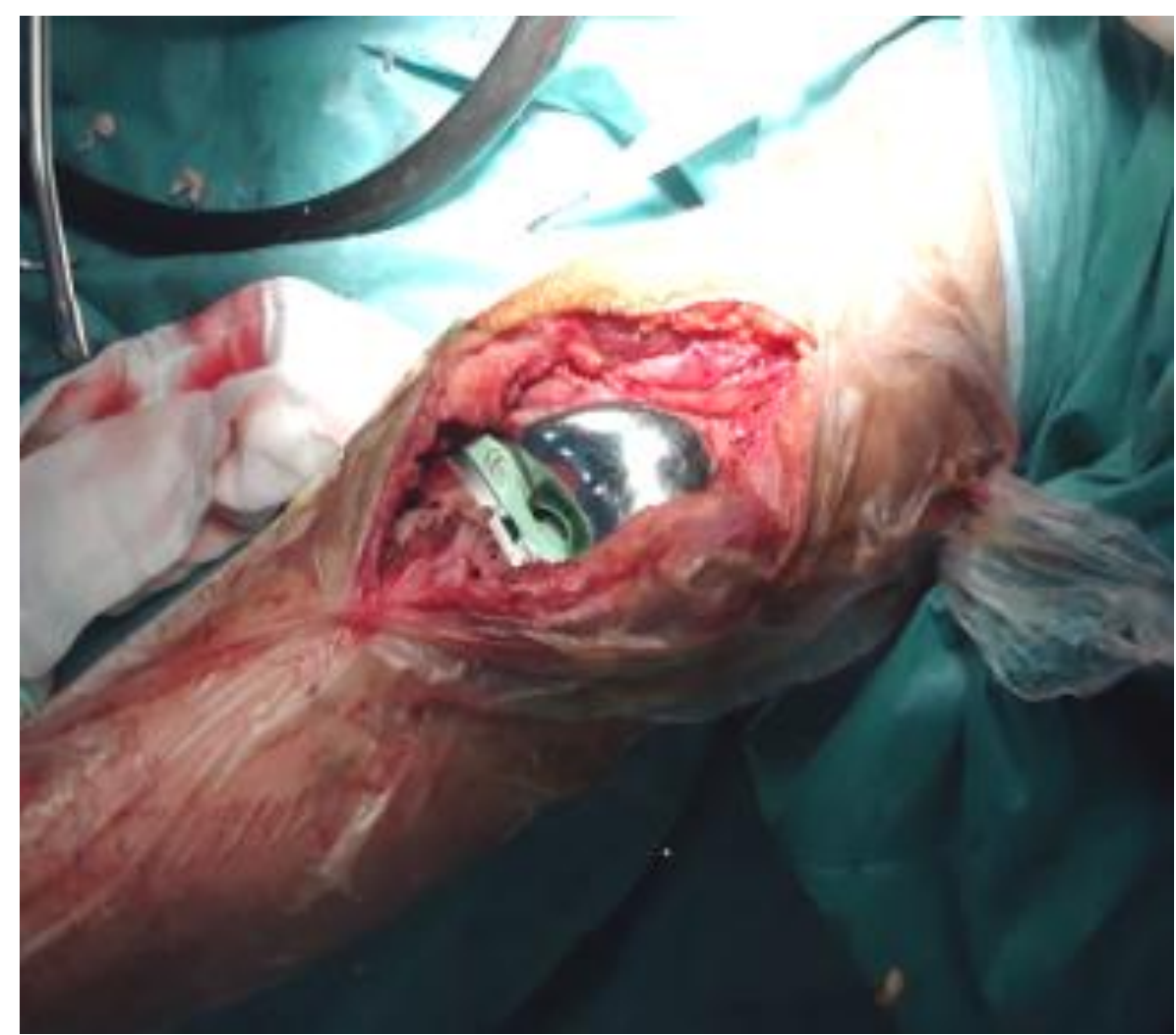

Figure (13): Insertion of final tibial and femoral component with tibial insert trial and knee extended until cement become hard.

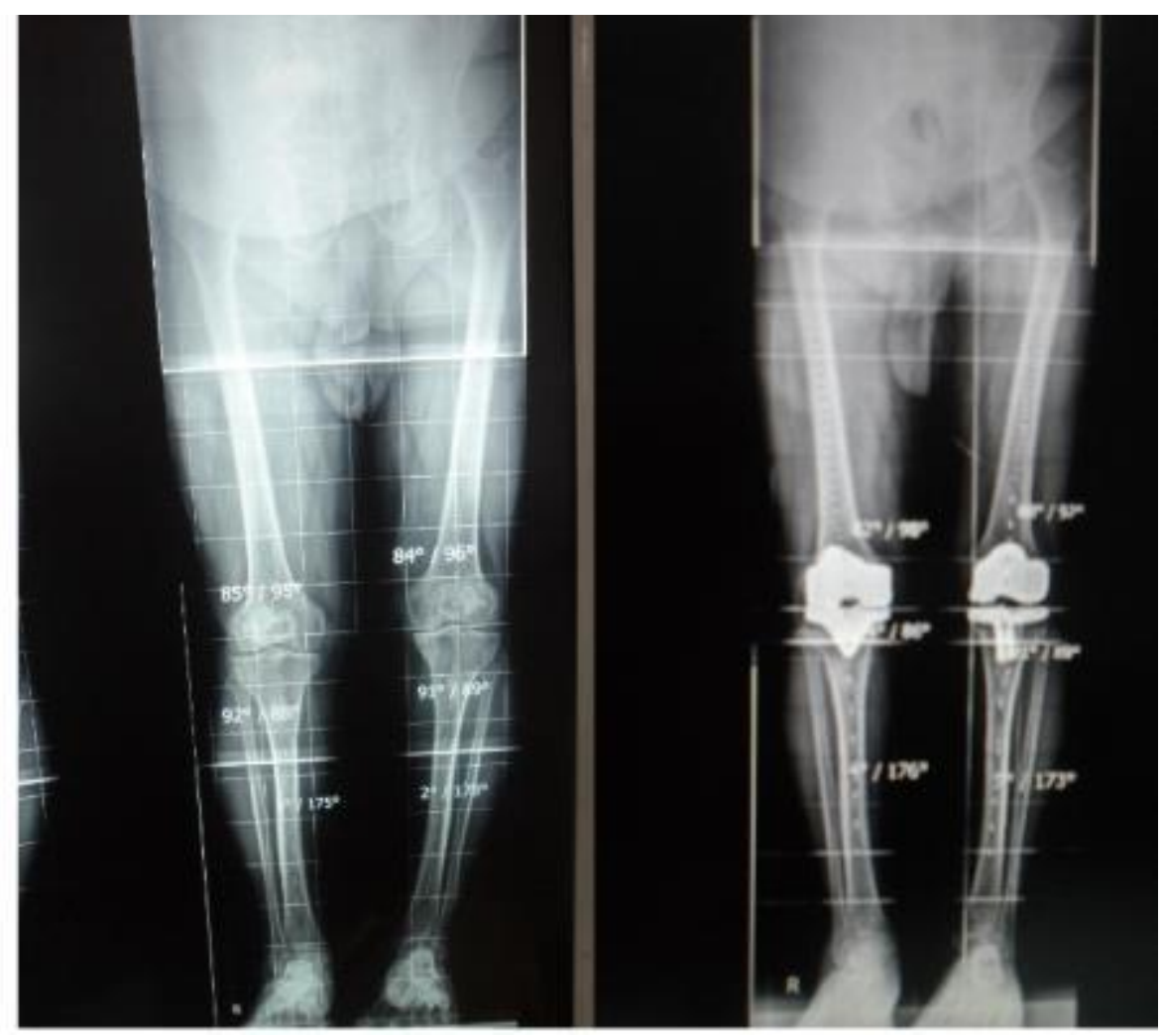

Figure (14): Standing long-leg X-rays pre-and postoperatively

Postoperative rehabilitation started from the second postoperative day. Standing long-leg X-rays were obtained for all patients 6 weeks postoperatively Figure (14). Knee society score (KSS) was assessed 6 months postoperatively and then every year for all patients.

The radiographic measurements included hip-knee-ankle angle (HKA, ideal 1 \pm 3 ), proximal tibial mechanical angle (PTMA, ideal $0 \pm 3$ ) and lateral distal femoral mechanical angle (LFMA, ideal $0 \pm 3$ ). 
Ethical approval and written informed consent: An approval of the study was obtained from Al-Azhar University academic and ethical committee. Every patient signed an informed written consent for acceptance of the operation.

\section{Statistical analysis}

Comparing pre-and postoperative alignment and KSS was performed using SPSS version 16-software package under Windows 7 operating system. Categorical data parameters were presented in the form of frequency and percent. Quantitative data were expressed in the form of mean $\pm \mathrm{SD}$. Comparison was performed by Kai square test for categorical data and independent and paired sample t-test for quantitative data.

\section{RESULTS}

All operations were successfully performed using PSI without resorting to the conventional technique. There was significant reduction in the mean HKA from 4.70 varus (SD 6.48) preoperatively to 1.27 varus (SD 4.8) postoperatively (P value 0.0000).The number of outliers decreased significantly as shown in tables (1) and (2). The mean postoperative LDFA and PTMA were found to be 89.3 and 87.9 respectively as shown in table (3). The mean knee society score had markedly increased from 31.2 (SD15.8) preoperatively to 85.3 (SD 11.4) postoperatively ( $\mathrm{P}$ value 0.000 ). 95\% of cases showed fair to excellent KSS (Table 4).

Table (1): Pre-and postoperative hip knee ankle (HKA) (Neutral: 3 to 3).

\begin{tabular}{|l|c|c|c|c|}
\hline \multirow{2}{*}{$\begin{array}{c}\text { Hip-Knee-ankle } \\
\text { (HKA) }\end{array}$} & \multicolumn{2}{|c|}{ Preoperative } & \multicolumn{2}{c|}{ Postoperative } \\
\cline { 2 - 5 } & No. & \% & 21 & \% \\
\hline Neutral & 6 & 15 & 19 & 47.5 \\
\hline Outlier & 34 & 85 & & \\
\hline P value & $\mathbf{0 . 0 0 1}$ & & & \\
\hline$X^{2}$ & $\mathbf{1 1 . 6}$ & & & \\
\hline Sig. & $* *$ & & & \\
\hline
\end{tabular}

Chi square was used to test the significance between groups $\quad * *$ Significant $(\mathrm{P}<0.01)$

Table (2): Pre-and postoperative HKA (Neutral: 4 to 4).

\begin{tabular}{|l|c|c|c|c|}
\hline \multirow{2}{*}{$\begin{array}{c}\text { Hip-Knee-ankle } \\
\text { (HKA) }\end{array}$} & \multicolumn{2}{|c|}{ Preoperative } & \multicolumn{2}{c|}{ Postoperative } \\
\cline { 2 - 5 } & No. & \% & No. & \% \\
\hline Neutral & 10 & 25 & 26 & 35 \\
\hline Outlier & 30 & 75 & & \\
\hline P value & $\mathbf{0 . 0 0 0}$ & & & \\
\hline $\mathrm{X}^{2}$ & $\mathbf{1 3 . 4}$ & & & \\
\hline Sig. & $* *$ & & & \\
\hline
\end{tabular}

Chi square was used to test the significance between groups $\quad * *$ Significant $(\mathrm{P}<0.01)$

Table (3): Lateral distal femoral mechanical angle and proximal tibial mechanical angle.

\begin{tabular}{|l|c|c|c|c|}
\hline \multirow{2}{*}{} & \multicolumn{2}{|c|}{$\begin{array}{c}\text { Lateral distal femoral mechanical angle } \\
\text { (LDFA) }\end{array}$} & \multicolumn{2}{c|}{$\begin{array}{c}\text { Proximal tibial mechanical angle } \\
\text { (PTMA) }\end{array}$} \\
\cline { 2 - 5 } & No. & \% & No. & \% \\
\hline Normal $\left(90^{\circ} \pm 3^{\circ}\right)$ & 27 & 67.5 & 23 & 57.5 \\
\hline abnormal & 13 & 32.5 & 17 & 42.5 \\
\hline Mean \pm SD & 89.3 & 3.9 & 87.9 & 3.5 \\
\hline
\end{tabular}

Chi square was used to test the significance between groups $\quad * *$ Significant $(\mathrm{P}<0.01)$

Table (4): Knee society score pre-and postoperative.

\begin{tabular}{|l|c|c|c|c|}
\hline \multirow{2}{*}{ Knee society score (KSS) } & \multicolumn{2}{c|}{ Preoperative } & \multicolumn{2}{c|}{ Postoperative } \\
\cline { 2 - 5 } & No. & $\mathbf{\%}$ & 2 & No. \\
\hline Poor (less than 60) & 37 & 93.5 & 3 & 7.5 \\
\hline Fair (61:69) & 3 & 7.5 & 3 & 7.5 \\
\hline Good (70:79) & 0 & 0 & 32 & 80 \\
\hline Excellent (More than 80) & 0 & 0 & & \\
\hline P value & $\mathbf{0 . 0 0 0}$ & & & \\
\hline $\mathrm{X}^{2}$ & $\mathbf{6 2 . 4}$ & & & \\
\hline Sig. & $* *$ & & \\
\hline
\end{tabular}




\section{DISCUSSION}

In this series, we assessed the results of PSI in terms of radiographic and clinical outcomes in a period of 3 years duration. We used CT scanning for surgical planning and not MRI to avoid extra cost and prolonged waiting list. Meanwhile, CT-based PSI has the advantage of easier planning, which can be done by the surgeon himself being not as sophisticated as MRI. Planning by the surgeon made the complete surgical technique under the control of the surgeon and not the technician.

Bone cuts in PSI cutting technique were done without any intramedullary guide, and this could probably decease the blood loss, rate of fat embolism, infection and bone injury. Moreover, surgical steps had markedly decreased in response to decreasing the number of used instruments into two cutting guides and box osteotome for posterior stabilizing knees and trial components.

It is noticed that there was marked improvement in the overall alignment, and the percentage of outliers decreased from $86 \%$ to $47 \%$. Vincent et al. ${ }^{(6)}$ followed some previous studies comparing postoperative coronal outliers between navigation and conventional TKR. They found that percentage of outliers in navigation was between $0 \%$ and $52 \%$ while in conventional technique it was between $2 \%$ and $72 \%$. In comparison to our study, we found the percentage of outliers was equal or higher than that of Vincent et al.'s for navigation but lower than that for conventional.

Ryan et al. ${ }^{(7)}$ compared PSI in restoring mechanical axis/kinematic axes to conventional with no statistically significant difference. They reported that PSI had significantly higher percentage of outliers in terms of restoring kinematic axis. Another study compared 57 patients undergoing TKR using PSI to the same number using conventional TKR with no statistically significant difference regarding postoperative coronal alignment ${ }^{[8]}$. On the contrary, several studies had documented better postoperative alignment after PSI compared to navigation and conventional techniques ${ }^{[6,9,10}$, and 11] .

Thienpont et al. ${ }^{(12)}$ did a systematic review and meta-analysis on 8 randomized control trials and 8 cohort studies comparing postoperative coronal mechanical alignment as well as coronal, axial and sagittal positions of tibial and femoral components. They found no statistically significant difference between PSI and conventional techniques. However, it was found that conventional was statistically better in tibial components' position (coronal, sagittal and axial) while PSI was better in coronal alignment of femoral components where PSI did not lead to better alignment than conventional.

Although PSI involves preoperative computer planning, there is mixed results about its superiority than conventional regarding alignment parameters. The postoperative alignment depends on many factors including soft tissue balance, deviation of saw during bone cuts, deviation during implantation of cementing components and extra-articular deformities such as bowing of tibia and femur

Regarding clinical assessment and function score, we found that there was marked improvement in KSS after 3 year with good to excellent results in more than $90 \%$ of knees regardless of the degree of mechanical alignment. All PSI procedures done in this work used PSI as cutting guides, while most of work in literature had described PSI as pin locator. Limitations of this study included lacking a control group for conventional cases or an assessment of rotational alignment of components by doing postoperative CT.

Although PSI has the same results of conventional technique in terms of axial alignment and functional scoring it is still better in terms of saving operative time, avoid medullary penetration and concurrent complications such as fat embolism, blood loss and infection.

\section{CONCLUSION}

PSI TKR is an effective technique in knee replacement as conventional method with the advantages of reduction of the operative steps and concurrent potential complications.

\section{REFERENCES}

1. Krishnan S, Dawood A, Richardsand A et al. (2012): A review of rapid prototyped surgical guidesfor patient-specific total knee replacemet. J Bone Joint Surg Br., 94 (11): 1457-61.

2. Hafez M (2012): Custom-made guides for Total Knee Arthroplasty. Insall Scott $\left(5^{\text {th }}\right.$ ed), Surgery of the knee, New York, Churchill Livingstone, Pp: 587-696.

3. Hafez M, Chelule K, Seedhom B et al. (2006): Computer-assisted total knee arthroplasty using patient-specific templating. Clin Orthop Relat Res., 444: 184-92

4. Michael P, Nam D, Haas $S$ (2012): Patient-Specific Instrumentation for Total Knee Arthroplasty. A Review Orthop Clin N Am., 43 (5): 17-22.

5. Sassoon A, Nam D, Nunley R et al. (2015): Systematic Review of Patient-specific Instrumentation in Total Knee Arthroplasty: New but Not Improved. Clin Orthop Relat Res., 473 (1): 151-8.

6. Vincent Y, Jeffrey H, Keith R et al. (2012): Lombardi Improved Accuracy of Alignment with Patient-specific Positioning Guides Compared with Manual Instrumentation in TKA. Clin Orthop Relat Res., 470 (1): 99-107.

7. Ryan M, Bradley S, Jinjun $\mathrm{Z}$ et al. (2012): Do Patient-specific Guides Improve Coronal Alignment in Total Knee Arthroplasty? Clin Orthop Relat Res., 470 (3): 895-902.

8. Ryan M, Bradley S, Erin L et al. (2012): Are Patient-specific Cutting Blocks Cost-effective for Total KneeArthroplasty? Clin Orthop Relat Res., 84 (2): 90-98.

9. Kiriakos D, Carsten O (2013): Frontal plane alignment after total knee arthroplasty using patient-specific instruments. International Orthopaedics (SICOT), 37 (1): 45-50.

10. Naeder H, Mai L, Stefanie P et al. (2014): Accuracy of Patient Specific Cutting Blocks in Total Knee Arthroplasty. https://www.hindawi.com/journals/bmri/2014/562919/

11. Koch P, Muller D, Pisan $M$ et al. (2013): Radiographic accuracy in TKA with a CT-based patient-specific cutting block technique. Knee Surg Sports Traumatol Arthrosc., 21 (10): 2200-5.

12. Thienpont E, Schwab P, Fennema $P$ (2014): A systematic review and meta-analysis of patient-specific instrumentation for improving alignment of the components in total knee replacement Bone Joint J., 96 (8): 1052-61. 